\title{
Radioactive Iodine Uptake in Response to Recombinant Human Thyrotropin Stimulation for Thyroid Remnant Ablation in Patients with Differentiated Thyroid Cancer
}

\author{
Koutsikos Johnn ${ }^{1,2 *}$, Velidaki Antigoni ${ }^{3}$, Angelidis George ${ }^{1}$, Kalkinis Antonios ${ }^{4}$ and Boutzios \\ Georgios $^{5}$ \\ ${ }^{1}$ Department of Nuclear Medicine, Army Share Fund Hospital, Athens, Greece \\ ${ }^{2}$ Department of Nuclear Medicine, Henry Dunant Hospital Center, Athens, Greece \\ ${ }^{3}$ Department of Nuclear Medicine, "Laikon" General Hospital, Athens, Greece \\ ${ }^{4}$ Department of Nuclear Medicine, Metropolitan Hospital, Athens, Greece \\ ${ }^{5}$ Endocrine Unit, Department of Pathophysiology, Medical School, National \& Kapodistrian University of Athens, "Laikon" General Hos- \\ pital, Athens, Greece
}

*Corresponding author: Koutsikos John, Department of Nuclear Medicine, Army Share Fund Hospital (417 NIMTS) Athens, Greece

\section{ARTICLE INFO}

Received: 幽 August 08, 2019

Published: 豐 August 20, 2019

Citation: Koutsikos J, Velidaki A, Angelidis G, Kalkinis A, Boutzios G. Radioactive Iodine Uptake in Response to Recombinant Human Thyrotropin Stimulation for Thyroid Remnant Ablation in Patients with Differentiated Thyroid Cancer. Biomed J Sci \& Tech Res 20(4)-2019. BJSTR. MS.ID.003488.

Keywords: Ablation; Iodine; rhTSH; Thyroid Cancer; Uptake

\section{ABSTRACT}

Purpose: Thyroid hormone withdrawal (THW) is required for the preparation of differentiated thyroid cancer (DTC) patients referred for thyroid remnant ablation. Alternatively, recombinant human thyrotropin (rhTSH) treatment may be performed. We aimed to evaluate whether pre-ablation radioiodine uptake (RAIU) measurement is affected by the performance of rhTSH preparation, particularly with regard to the outcome of the ablation therapy.

Methods: We retrospectively studied 139 DTC patients (117 females, mean age 46.3 years). Eighty-four patients discontinued thyroxine or triiodothyronine treatment (group A), whereas 55 received 2 doses of rhTSH, without THW (group B). Pre-ablation RAIU values were recorded for all patients. In patients with RAIU $>9 \%$, a lower RAI activity was administered. Patients were followed up based on whole-body imaging and stimulated thyroglobulin levels in 12-18 months post-ablation.

Results: Mean RAIU value for group A was $2.41 \%$ and for group B 1.53\%. Eight group A patients had RAIU measurement $>9 \%$. In total, $123 / 139$ patients found to be diseasefree in the follow-up; 73/84 group A and 50/55 group B patients (ablation rates $86.9 \%$ and $90.9 \%$, respectively).

Conclusion: rhTSH preparation was associated with RAIU underestimation in DTC patients. However, the management of these patients was not influenced by the recorded variations in RAIU values.

\section{Introduction}

The vast majority of differentiated thyroid tumours arise from thyroid follicular epithelial cells; papillary cancer corresponds to approximately $85 \%$ of cases, whereas about $12 \%$ of tumours have follicular histology, including Hürthle cell carcinomas [1]. Initial therapeutic management aims to remove the primary tumour with as low surgery-related morbidity as possible [2]. Total thyroidectomy (TT) has been indicated as the primary surgical treatment option for nearly all differentiated thyroid lesions $>1 \mathrm{~cm}$, independently of the presence of loco-regional or distant metastases [3]. When performing TT, the surgeon aims to remove as much thyroid tissue within the operative bed as possible. However, in a significant number of patients, a small amount of thyroid tissue may remain. Complete thyroid tissue removal is demonstrated based on a negative post-operative whole-body scan (TxWBS), radioactive iodine uptake (RAIU) $<1 \%$, and undetectable levels of thyroglobulin (Tg). In a large study sample, Salvatori et 
al. demonstrated the presence of thyroid remnant (TR) in $93.1 \%$ of the patients who underwent TT due to differentiated thyroid cancer (DTC) [4]. Incomplete thyroid tissue removal may lead to the performance of radioactive iodine (RAI) therapy for TR ablation $[5,6]$.

In general, RAIU measurements provide valuable information for the therapeutic management of DTC patients. Residual RAIavid disease can be detected, and TR uptake is evaluated through RAIU testing. As previously mentioned, RAIU data are employed, in combination with TxWBS findings and Tg levels, in the decisionmaking process regarding the need for RAI ablation. Interestingly, Holsinger et al. reported that post-operative RAIU $<0.2 \%$ is associated with the highest likelihood of undetectable Tg levels after TT, resulting in an even smaller need for TR ablation [7]. Moreover, Zerva et al. reported that the ratio of Tg levels to RAIU values, during the immediate post-surgical period, can be used as a tool for the identification of patients with metastatic disease [8]. In these patients, higher initial RAI therapy doses may be selectively administered in order to obtain a better outcome. On the other hand, the tailoring of RAI activities based on RAIU values was not found more advantageous compared to the fixing dosing, in terms of the success of TR ablation [9-11]. A dose of approximately $1,110 \mathrm{MBq}(30 \mathrm{mCi})$ is generally favoured over higher administered activities that may be considered only if a large TR is suspected [5]

In DTC patients considered to be at low risk of recurrence or mortality, previous management guidelines advocated selective performance of RAI ablation, due to its side-effects $[3,6,12,13]$. More recently, according to the American Thyroid Association (ATA) guidelines task force on thyroid nodules and DTC, TT may be performed in patients with tumour size $1-4 \mathrm{~cm}$, but it is strongly indicated only in patients with tumour $>4 \mathrm{~cm}$, or gross extrathyroidal extension, or clinically apparent metastatic disease [5]. These recommendations are formulated in international centres of excellence; however, they may not be fully applicable in other institutions where TT remains the primary surgical procedure in DTC patients [14-16]. Therefore, especially in countries with few specialized thyroid cancer centres, such as Greece, RAIU measurements continue to have a significant role in making therapeutic decisions, in most patients with regard to the need for RAI ablation.

A goal thyroid-stimulating hormone (TSH) concentration of $\geq 30 \mathrm{mIU} / \mathrm{L}$ is generally adopted in RAI therapy preparation, since TSH levels above this threshold are required for incompletely resected thyroid tissue to significantly concentrate 131I [17]. According to the recent ATA guidelines, preparation with recombinant human thyrotropin (rhTSH) stimulation is an acceptable alternative to thyroid hormone withdrawal (THW) for achieving TR ablation in ATA low-risk DTC patients, ATA intermediate-risk DTC patients, especially in those without extensive lymph node involvement, and DTC patients of any risk level with significant co-morbidity that may preclude THW prior to RAI administration. 5 rhTSH preparation is also advocated for attaining TSH elevation in DTC patients, referred for RAI ablation, based on the European Association of Nuclear Medicine (EANM) guidelines [6]. In the present study, we aimed to investigate the presence of variations in RAIU measurements after either rhTSH preparation or THW in DTC patients referred for TR ablation, and to examine whether these potential discrepancies could influence the efficacy of TR ablation.

\section{Methods}

The influence of rhTSH preparation on RAIU measurements was retrospectively investigated in patients with DTC referred for TR ablation to the Department of Nuclear Medicine, Bioclinic General Hospital in Piraeus, Greece. All patients who underwent this procedure between January 2013 and December 2015 were considered as potential participants. Exclusion criteria included

a. Histological component of low differentiated thyroid carcinoma

b. Established metastatic disease (M1),

c. Patients presenting with incomplete histopathological data,

d. Locoregional tissue invasion or macroscopic extrathyroidal extension,

e. Patients who had not (at least) undergone central neck lymph node dissection, and

f. Patients who missed follow-up appointments. Based on these criteria, 134 patients were enrolled in the study (Table 1).

Table 1: Patient Characteristics.

\begin{tabular}{|c|c|c|c|}
\hline & Study Sample & Group A & Group B \\
\hline Patient number $(\mathrm{n})$ & 139 & 84 & 55 \\
\hline Mean age (years) & $46.3+/-13.3$ & $45.1+/-12.9$ & $50.5+/-13.7$ \\
\hline Males & 22 & 13 & 9 \\
\hline Females & 117 & 71 & 46 \\
\hline Tumour size $\leq 1 \mathrm{~cm}$ & 71 & 44 & 27 \\
\hline Tumour size $>1 \mathrm{~cm}$ & 68 & 40 & 28 \\
\hline METE & 104 & 67 & 37 \\
\hline Multifocality & 56 & 34 & 22 \\
\hline Bilaterality & 46 & 27 & 19 \\
\hline Node metastasis & 56 & 36 & 20 \\
\hline
\end{tabular}

Note: METE: microscopic extra-thyroidal extension.

Group A: thyroid hormone withdrawal group.

Group B: recombinant human thyrotropin treatment group.

The sample consisted of 117 females and 22 males (age 46.3+/13.3 years, range 20-82). Thyroxine or triiodothyronine treatment was discontinued in 84 patients (group A), whereas two rhTSH doses were administered in 55 patients without THW (group B). 
Serum TSH levels were measured prior to RAI administration in order to evaluate the degree of hormone elevation, requiring TSH levels in excess of $30 \mathrm{mIU} / \mathrm{L}$. The measurements were performed using immunoradiometric assays (IRMA, Immunotech, Beckman Coulter co., Prague Czech Rep.) with a sensitivity of $0.025 \mathrm{IU} / \mathrm{mL}$. Serum Tg levels were measured by immunoradiometric assays (IRMA, Immunotech, Beckman Coulter co., Prague, Czech Rep) with a sensitivity of $1 \mathrm{ng} / \mathrm{mL}$. Antithyroglobulin antibodies were measured by IRMA (Immunotech, Beckman Coulter co., Prague, Czech Rep.) with an analytical sensitivity of $5 \mathrm{IU} / \mathrm{mL}$.

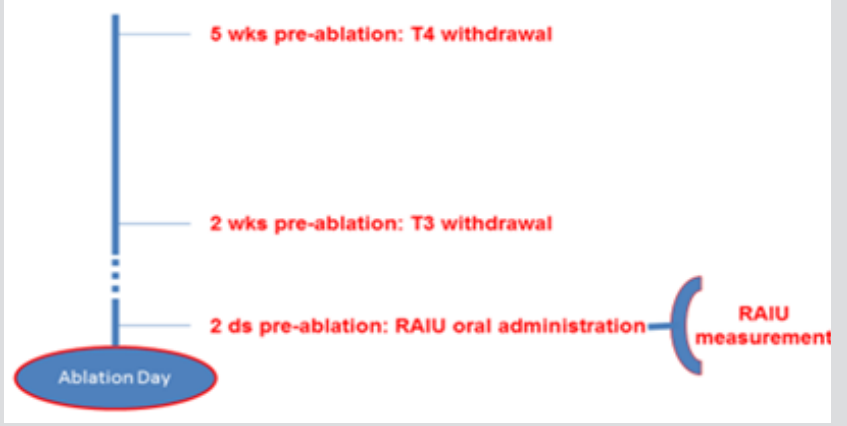

Figure 1: Thyroid hormone withdrawal (group A). RAIU measurement was performed 2 days prior ablation, following an oral 131I administration of $60-80 \mu \mathrm{Ci}$. RAIU: radioiodine uptake.

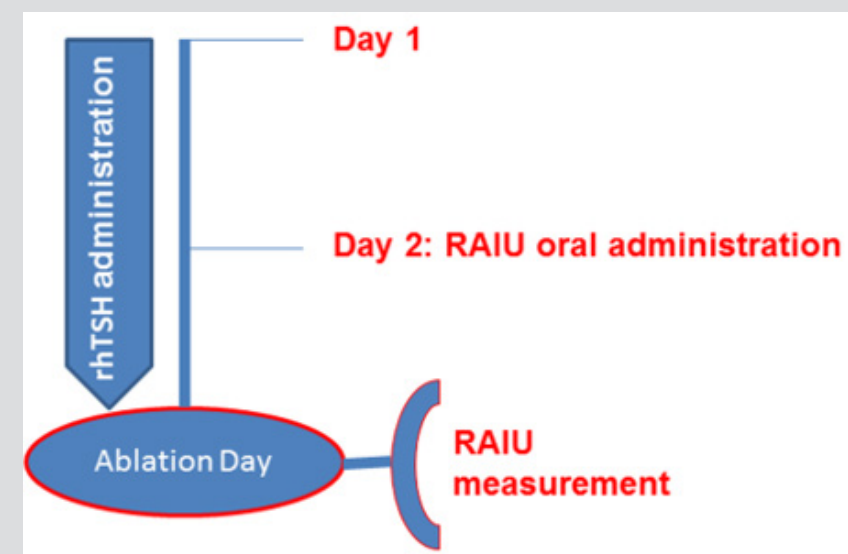

Figure 2: Recombinant human (rh) thyrotropin treatment (group B). RAIU measurement was performed at the day of ablation, 1 day after RAIU oral administration. RAIU: radioiodine uptake.

All patients started a low-iodine diet 2 weeks before RAI administration. In group A patients, thyroxine was withdrawn for 5 weeks prior to RAI treatment and was substituted by triiodothyronine administration during the first 3 weeks of thyroxine withdrawal. Triiodothyronine was withdrawn for at least 2 weeks before RAI therapy. RAIU was performed 2 days prior ablation, after an oral 131I administration of 2,220-2,960 MBq (60$80 \mu \mathrm{Ci}$ ) (Figure 1). In group B patients, 131I was administered at the second day of rhTSH administration and RAIU was performed $24 \mathrm{~h}$ later, at the day of ablation (Figure 2). The pre-treatment RAIU value in the neck region was assessed using a standard collimated probe. This probe measures the percentage of a dose in the thyroid bed with the subtraction of a neck equivalent background level measured over the patient's thigh, in comparison to a standard of equal value to the administered dose. Patients received 30-120 mCi of 131I at the day of ablation. Particularly, a dose of 30-40 mCi was administered to patients with RAIU $>9.0 \%$ and no evidence of RAIavid tissue outside the thyroid bed, in order to avoid potential sideeffects associated with higher activities. Tg levels were recorded in all participants on the day of RAIU measurements. Successful ablation was defined as no visible or faint uptake on the followup WBS after 12-18 months, in combination with undetectable stimulated Tg levels.

\section{Results}

The mean age of patients treated under THW (group A) was $45.1+/-12.9$ years, whereas the mean age of rhTSH-prepared patients (group $\mathrm{B}$ ) was $50.5+/-13.7$ years $(\mathrm{P}=0.1)$. The number of female patients was higher but similar in both groups ( $85 \%$ in group A and $84 \%$ in group B). No statistically significant differences were observed between the two groups regarding administered dose and retained activity. Histologically, all patients suffered from papillary thyroid cancer. Table 1 summarizes information for the sample, group A and group B patients. In group A, mean RAIU measurement was $2.41+/-2.63 \%$ (range $0.1-14.3 \%$ ), whereas the corresponding value for rhTSH-prepared patients was $1.53+/-1.30 \%$ (range 0.2 6.3\%) ( $\mathrm{P}=0.01$ ) (Figure 3). RAIU values over 9\% were observed in eight group A patients. Mean Tg concentration was 4.84+/-8.29 $\mathrm{ng} / \mathrm{mL}$ (range $0.1-48.3 \mathrm{nh} / \mathrm{mL}$ ) in group A patients and 6.31+/$6.79 \mathrm{ng} / \mathrm{mL}$ (range 0.1-32.6) in group $\mathrm{B}$ patients $(\mathrm{P}=0.27)$ on the day of RAIU measurement (all patients with TSH $>30 \mathrm{ng} / \mathrm{mL}$ ). RAI administered activity was $92.2+/-23,3 \mathrm{mCi}$ in group A patients and $93.1+/-25.5 \mathrm{mCi}$ in group $\mathrm{B}$ patients $(\mathrm{P}=0.57)$. Patients showing RAIU $>9 \%$ received a smaller RAI dose of 34+/-5.5 mCi (Table 2). Moreover, these eight patients underwent a second RAI treatment. There was no other patient receiving second RAI therapy, either in group A or B. After 12-18 months, 88.5\% (123/139) of patients were found to be disease free; successful ablation was demonstrated in $86.9 \%$ (73/84) of group A patients and in 90.9\% (50/55) of group B patients $(\mathrm{P}=$ n.s. $)$.

Table 2: Mean administered activity (AA) in group A and group $B$ patients with respect to the radioiodine uptake (RAIU) values.

\begin{tabular}{|c|c|c|c|c|}
\hline & Group A & & Group B & \\
\hline & RAIU <9\% & RAIU $>9 \%$ & RAIU <9\% & RAIU $>9 \%$ \\
\hline $\begin{array}{c}\text { Number of } \\
\text { patients (n) }\end{array}$ & 76 & 8 & 55 & 0 \\
\hline $\begin{array}{c}\text { Mean AA } \\
(\mathrm{mCi})\end{array}$ & $92.2+/-23.3$ & $34+/-5.5$ & $\begin{array}{c}93.1+/- \\
25.5\end{array}$ & - \\
\hline
\end{tabular}


Note: Group A: thyroid hormone withdrawal group.

Group B: recombinant human thyrotropin treatment group.

\section{Discussion}

In the present study we investigated the potential effects of rhTSH preparation on pre-ablation RAIU measurements. According to our results, thyroid bed RAIU may be underestimated in rhTSHprepared patients. Mean RAIU values were $2.41+/-2.63 \%$ and $1.53+/-1.30 \%$ for group A and group B, respectively. This finding may be associated with the faster RAI clearance under euthyroid state [18]. Notably, the observed variations did not seem to affect negatively the management of patients enrolled in the study. Follow-up results after 12-18 months revealed high ablation rates both in group A (86.9\%) and group B patients (90.9\%). Moreover, serum TSH and Tg values on the day of RAIU measurements could be an estimate of TR size. 5 However, no significant differences regarding these variables were observed between group $\mathrm{A}$ and group B patients, implying no significant differences in TR size among the two groups.

Although neck ultrasonography and 99mTc04 - thyroid scintigraphy are routinely used for the therapeutic decision making in DTC patients, these modalities may provide minimal information with respect to TR estimation. Neither neck ultrasonography nor $99 \mathrm{mTc0} 4$ - thyroid scintigraphy should be used for thyroid remnant estimation [19]. On the other hand, TR RAIU can serve as a valuable tool for the evaluation of the completeness of resection and the prediction of individual recurrence risk [20]. Moreover, pretreatment measurement of thyroid bed RAIU contributes not only to the therapeutic management regarding the decision to either perform TR ablation or not, but also to the selection of RAI activity when tailoring dose according to RAIU values. This test may clearly maximise the degree of individualisation in therapeutic decisionmaking after TT, an advantage especially important in paediatric patients. 6 Consequently, it is a useful tool, particularly in cases of suboptimal TR extent evaluation based on the surgical report or neck ultrasonography [21].

Traditionally, the decision regarding the administered activity for TR ablation could be made based on three different approaches:

\section{a. Empirical fixed doses.}

b. Upper bound limits according to blood and whole-body dosimetry.

\section{c. Quantitative tumour dosimetry [8].}

TR ablation can be performed successfully with RAI doses based on TxWBS neck uptake, although there are published data supporting a fixed-dose protocol.9,21 Nevertheless, RAIU-guided strategy has undoubtedly two important advantages over fixeddose protocols; unnecessary radiation exposure can be avoided and the occurrence of local side effects due to RAI administration is lower [22-24]. In our department, if RAIU is over $9.0 \%$ and there is no evidence of RAI-avid tissue outside the thyroid bed, we opt for lower activities of 30-40 $\mathrm{mCi}$, instead of doses ranging between 80-120 mCi. In the present study eight group A patients had RAIU values $>9 \%$, indicating a higher risk of side-effects in the postablation period. RAI therapy is generally safe; however, there is a risk for potential side-effects $[25,26]$.

In general, TSH levels $\geq 30 \mathrm{mUI} / \mathrm{L}$ are required for successful TR ablation [17] Thyrocytes up-regulate the expression, transcription and activity of sodium/iodine symporter in response to endogenous TSH stimulation. For this reason, THW is commonly used to optimize RAI retention, and TSH stimulation is believed to increase RAI uptake in a dose-dependent and a time-dependent manner. Nevertheless, THW is also associated with the hypothyroidism morbidity and impaired quality of life during the preparatory phase of RAI therapy. The availability of rhTSH provides a different approach in enhancing serum TSH concentration, without inducing hypothyroidism. rhTSH preparation is considered as an alternative method to THW based on both ATA and EANM guidelines.5,6 Similarly, according to a 2015 position statement of the Italian Society of Endocrinology, DTC patients referred for TR ablation should be prepared by rhTSH administration; THW may be considered in certain cases where rhTSH is not available or not affordable [27].

The efficacy of rhTSH-aided RAI therapy for TR ablation has been evaluated in several studies. It was reported to be equally effective to THW in recent meta-analyses [28,29]. In our study, ablation rates were optimal, both in rhTSH-prepared patients and in patients treated under hypothyroid state. Important advantages of rhTSH preparation have been reported in terms of quality of life and adverse effects during and after ablation, including fatigue, headache, sialadenitis, neck pain, taste loss, nausea, radiation gastritis, and bone pain.12 [30-32], Notably, rhTSH-aided TR ablation was linked to decreased irradiation to the patients. In particular, the specific absorbed dose to the blood was significantly lower in patients undergoing rhTSH preparation compared to those randomized to the THW group [33]. Lower red marrow absorbed dose was also reported [34]. Although rhTSH preparation significantly increases the overall cost of the treatment, the balance may be in favour of its use when the cost to the society due to hypothyroidism - associated loss of productivity (days off work) is taken into account $[35,36]$. Moreover, rhTSH may allow a shorter hospitalization length because of the faster RAI excretion, partially compensating its high cost [35-37]. Thus, based on a cost-effectiveness analysis, rhTSH preparation should be considered "good value-for-money" with the benefits to both patients and the society obtained at modest net cost [38]. Similar findings were reported for a non-western patient population [39].

\section{Conclusion}

RAI ablation continues to have an important role in the management of DTC patients since TT remains the primary therapeutic option for patients suffering from DTC in many 
countries, particularly in those where specialized thyroid cancer services are rare. We suggest that the pre-ablation TR estimation is helpful whenever RAI therapy is performed in non-specialized clinics. RAIU measurement represents a valuable tool for the assessment of the need for ablation, including the appropriate RAI activity. Based on our protocol, RAIU test is applicable even after rhTSH preparation which permits the performance of RAI therapy without the THW-related adverse effects. Although we showed that RAIU may be underestimated in rhTSH-prepared patients, compared to patients who underwent THW, the observed variations did not affect negatively the patient management; therefore, these variations were not found to influence the outcome of ablation therapy. Finally, our findings were in accordance to the previously published studies confirming the efficacy of RAI therapy after rhTSH preparation.

\section{Reference}

1. Aschebrook Kilfoy B, Ward MH, Sabra MM, Devesa SS (2011) Thyroid cancer incidence patterns in the United States by histologic type, 19922006. Thyroid 21: 125-134.

2. Loyo M, Tufano RP, Gourin CG (2013) National trends in thyroid surgery and the effect of volume on short-term outcomes. Laryngoscope 123 2056-2063.

3. Cooper DS, Doherty GM, Haugen BR (2009) Revised American Thyroid Association management guidelines for patients with thyroid nodules and differentiated thyroid cancer. Thyroid 19: 1167-1214.

4. Salvatori M, Raffaelli M, Castaldi P (2007) Evaluation of the surgical completeness after total thyroidectomy for differentiated thyroid carcinoma. Eur J Surg Oncol 33: 648-654.

5. Haugen BR, Alexander EK, Bible KC (2016) 2015 American Thyroid Association Management Guidelines for Adult Patients with Thyroid Nodules and Differentiated Thyroid Cancer: The American Thyroid Association Guidelines Task Force on Thyroid Nodules and Differentiated Thyroid Cancer. Thyroid 26: 1-133.

6. Luster M, Clarke SE, Dietlein M (2008) Guidelines for radioiodine therapy of differentiated thyroid cancer. Eur J Nucl Med Mol Imaging 35: 1941-1959.

7. Holsinger FC, Ramaswamy U, Cabanillas ME (2014) Measuring the extent of total thyroidectomy for differentiated thyroid carcinoma using radioactive iodine imaging: relationship with serum thyroglobulin and clinical outcomes. JAMA Otolaryngol Head Neck Surg 140: 410-415.

8. Zerva B, Koutsikos J, Palestidis C, Kounadi E, Gerali S (2006) Value of thyroglobulin to 131I uptake ratio in selection of initial therapy dose of 131 in patients with differentiated thyroid carcinoma. Q J Nucl Med Mol Imaging 50: 348-354.

9. Verkooijen RB, Verburg FA, Van Isselt JW, Lips CJ, Smit JW, et al. (2008) The success rate of I-131 ablation in differentiated thyroid cancer: comparison of uptake-related and fixed-dose strategies. Eur J Endocrinol 159: 301-307.

10. Verburg FA, Verkooijen RB, Stokkel MP, Van Isselt JW (2009) The success of 131I ablation in thyroid cancer patients is significantly reduced after a diagnostic activity of 40 MBq 131I. Nuklearmedizin 48: 138-142.

11. Yap BK, Murby B (2014) No adverse effect in clinical outcome using low preablation diagnostic (131) I activity in differentiated thyroid cancer: refuting thyroid-stunning effect. J Clin Endocrinol Metab 99: 2433-2440.

12. Pacini F, Schlumberger M, Dralle H (2006) European consensus for the management of patients with differentiated thyroid carcinoma of the follicular epithelium. Eur J Endocrinol 154: 787-803.
13. Silberstein EB, Alavi A, Balon HR (2012) The SNMMI practice guideline for therapy of thyroid disease with 131I 3.0. J Nucl Med 53: 1633-1651.

14. Dietlein M, Verburg FA, Luster M, Reiners C, Pitoia F, et al. (2011) One should not just read what one believes: the nearly irresolvable issue of producing truly objective, evidence-based guidelines for the management of differentiated thyroid cancer. Eur J Nucl Med Mol Imaging 38: 793-798.

15. Huang TW, Lai JH, Wu MY, Chen SL, Wu CH, et al. (2013) Systematic review of clinical practice guidelines in the diagnosis and management of thyroid nodules and cancer. BMC Med 11: 191.

16. Frangos S, Iakovou IP, Marlowe RJ (2015) Difficulties in deciding whether to ablate patients with putatively "low-intermediate-risk" differentiated thyroid carcinoma: do guidelines mainly apply in the centres that produce them? Results of a retrospective, two-centre quality assurance study. Eur J Nucl Med Mol Imaging 42: 2045-2055.

17. Edmonds CJ, Hayes S, Kermode JC, Thompson BD (1977) Measurement of serum TSH and thyroid hormones in the management of treatment of thyroid carcinoma with radioiodine. Br J Radiol 50: 799-807.

18. Pacini F, Molinaro E, Castagna MG (2002) Ablation of thyroid residues with $30 \mathrm{mCi}$ (131) I: a comparison in thyroid cancer patients prepared with recombinant human TSH or thyroid hormone withdrawal. J Clin Endocrinol Metab 87: 4063-4068.

19. Liu G, Li N, Li X, Chen S, Du B, et al. (2016) Thyroid Remnant Estimation by Diagnostic Dose (131) I Scintigraphy or $(99 \mathrm{~m})$ Tc04(-) Scintigraphy after Thyroidectomy: A Comparison with Therapeutic Dose (131) I Imaging. Biomed Res Int 4763824.

20. Schneider DF, Ojomo KA, Chen H, Sippel RS (2013) Remnant uptake as a postoperative oncologic quality indicator. Thyroid 23: 1269-1276.

21. Zidan J, Hefer E, Iosilevski G (2004) Efficacy of I131 ablation therapy using different doses as determined by postoperative thyroid scan uptake in patients with differentiated thyroid cancer. Int J Radiat Oncol Biol Phys 59: 1330-1336.

22. Maxon HR, Englaro EE, Thomas SR (1992) Radioiodine-131 therapy for well-differentiated thyroid cancer--a quantitative radiation dosimetric approach: outcome and validation in 85 patients. J Nucl Med 33: 11321136.

23. Alexander C, Bader JB, Schaefer A, Finke C, Kirsch CM (1998) Intermediate and long-term side effects of high-dose radioiodine therapy for thyroid carcinoma. J Nucl Med 39: 1551-1554.

24. Burmeister LA, Du Cret RP, Mariash CN (1991) Local reactions to radioiodine in the treatment of thyroid cancer. Am J Med 90: 217-222.

25. Fard Esfahani A, Emami Ardekani A, Fallahi B (2014) Adverse effects of radioactive iodine-131 treatment for differentiated thyroid carcinoma. Nucl Med Commun 35: 808-817.

26. Lu L, Shan F, Li W, Lu H (2016) Short-Term Side Effects after Radioiodine Treatment in Patients with Differentiated Thyroid Cancer. Biomed Res Int 4376720.

27. Pacini F, Brianzoni E, Durante C (2016) Recommendations for postsurgical thyroid ablation in differentiated thyroid cancer: a 2015 position statement of the Italian Society of Endocrinology. J Endocrinol Invest 39: 341-347.

28. Pak K, Cheon GJ, Kang KW (2014) The effectiveness of recombinant human thyroid-stimulating hormone versus thyroid hormone withdrawal prior to radioiodine remnant ablation in thyroid cancer: a meta-analysis of randomized controlled trials. J Korean Med Sci 29: 811817.

29. Fu H, Ma C, Tang L, Wu F, Liu B, et al. (2015) Recombinant human thyrotropin versus thyroid hormone withdrawal in radioiodine remnant ablation for differentiated thyroid cancer: a meta-analysis. Q J Nucl Med Mol Imaging 59: 121-128.

30. Mallick U, Harmer C, Yap B (2012) Ablation with low-dose radioiodine and thyrotropin alfa in thyroid cancer. N Engl J Med 366: 1674-1685. 
31. Schlumberger M, Catargi B, Borget I (2012) Strategies of radioiodine ablation in patients with low-risk thyroid cancer. N Engl J Med 366: 1663-1667.

32. Iakovou I, Goulis DG, Tsinaslanidou Z, Giannoula E, Katsikaki G, et al. (2016) Effect of recombinant human thyroid-stimulating hormone or levothyroxine withdrawal on salivary gland dysfunction after radioactive iodine administration for thyroid remnant ablation. Head Neck 38: E227-230.

33. Hänscheid H, Lassmann M, Luster M (2006) Iodine biokinetics and dosimetry in radioiodine therapy of thyroid cancer: procedures and results of a prospective international controlled study of ablation after rhTSH or hormone withdrawal. J Nucl Med 47: 648-654.

34. Vaiano A, Claudio Traino A, Boni G (2007) Comparison between remnant and red-marrow absorbed dose in thyroid cancer patients submitted to 131I ablative therapy after rh-TSH stimulation versus hypothyroidism induced by L-thyroxine withdrawal. Nucl Med Commun 28: 215-223.

35. Borget I, Remy H, Chevalier J (2008) Length and cost of hospital stay

\section{ISSN: 2574-1241}

DOI: 10.26717/BJSTR.2019.20.003487

John Koutsikos. Biomed J Sci \& Tech Res

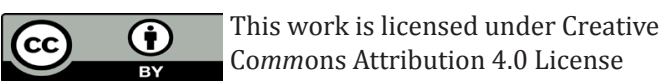

Submission Link: https://biomedres.us/submit-manuscript.php of radioiodine ablation in thyroid cancer patients: comparison between preparation with thyroid hormone withdrawal and thyrogen. Eur J Nucl Med Mol Imaging 35: 1457-1463.

36. Mernagh P, Suebwongpat A, Silverberg J, Weston A (2010) Costeffectiveness of using recombinant human thyroid-stimulating hormone before radioiodine ablation for thyroid cancer: the Canadian perspective. Value Health 13: 180-187.

37. Vallejo Casas JA Mena Bares LM, Gálvez MA, Marlowe RJ, Latre Romero JM, Martínez Paredes M (2011) Treatment room length-of-stay and patient throughput with radioiodine thyroid remnant ablation in differentiated thyroid cancer: comparison of thyroid-stimulating hormone stimulation methods. Nucl Med Commun 32: 840-846.

38. Mernagh P, Campbell S, Dietlein M, Luster M, Mazzaferri E, et al. (2006) Cost-effectiveness of using recombinant human TSH prior to radioiodine ablation for thyroid cancer, compared with treating patients in a hypothyroid state: the German perspective. Eur J Endocrinol 155: 405414.

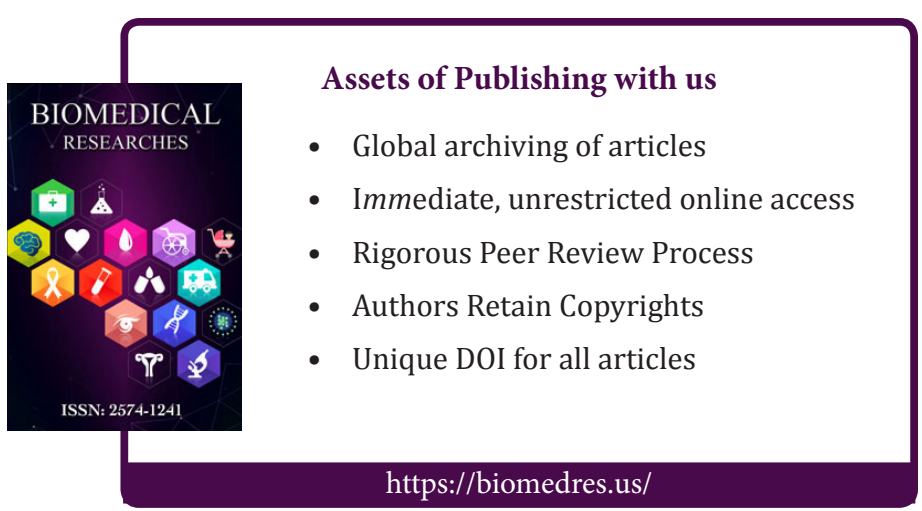

